\title{
Stochastic Simulation of Feeding Behavior of Abalone Haliotis discus hannai
}

\author{
Yasuhiro Hayakawa, ${ }^{* 1}$ Hiroshi Watanabe, ${ }^{* 2}$ and Jiro Kittaka*1
}

(Accepted February 6, 1987)

\begin{abstract}
Stochastic simulation of feeding behavior of abalones on algae in a restricted area was presented, based on a modified model for marine herbivorous feeding. Their feeding behavior was characterized by the stochastic processes of moving, feeding and resting. In the process of moving, vectors of the movement consisted of moving velocities and relative directions with probability density functions of log-normal and von Mises distribution, respectively, that were independent of each other. In the process of feeding and resting, hourly feeding rate and daily active duration the rest of which in a day was resting duration, were characterized by a bivariate normal distribution with a certain negative correlation coefficient with each other. Parameters in these processes were estimated, based upon observations of abalones in the rearing tanks. Resultant feeding process by this stochastic simulation was compared with that by another deterministic simulation and observed feeding of abalones upon young algae.
\end{abstract}

Many theoretical and experimental studies on optimal foraging for patchily-distributed prey of visual predators such as birds and so on, have successfully been made to show the development of deterministic continuous model by Charnovis and Krebs et al..$^{2)}$ into stochastic discrete model by Iwasa et al..3) The former is deterministic and continuous in that it is characterized by the time rate of continuous energy intake, or marginal capture rate, and the latter is stochastic and discrete in that it is based on the expectation of remaining prey distributed discretely in the patch.

As for the predators using chemical and tactile cues to detect and evaluate prey, it may take much more time for them to search preys and travel from one patch to another than the visual predator. As Elner and Hughes') reported on the maximization of time rate of energy intake by the shore crab foraging for mussels, predator's total handling time which includes the period of recognition, shell crushing and eating up played an important role in its choosing an optimal mussel size. However, studies on the details of searching and traveling processes have not yet been sufficient.

Time for random searching was taken into analytical consideration in the model stated above. ${ }^{3)}$ On the other hand, time for random traveling of small insects among their discretely-distributed habitats was studied numerically by Kitching ${ }^{5)}$ on the assumption that the probability density distribution of directions of movement was either a uniform or normal one on the basis of statistics of empirical data. In a similar fashion, time for random searching and constant feeding of marine herbivora in a restricted area was presented by Hayakawa and Kittaka ${ }^{\text {) }}$ in their model for feeding behavior of sea urchin upon algae. These kinds of models might be designated as a stochastic, discrete, and numerical model with statistical submodels governing the processes of concern, such as movement, feeding, and so on.

As a matter of fact, quantitative understanding of predator-prey systems has recently been of urgent and practical importance to scientific fisheries in coastal waters in Japan, particularly with regard to the increasing release of juvenile abalones cultured artificially from the hatchery into natural waters.

This paper presents a stochastic simulation of feeding behavior of abalone upon marine algae, based on the stochastic model ${ }^{\text {s) }}$ for marine herbivorous feeding with some additional modifications of the processes of moving, feeding and resting.

*1 School of Fisheries Sciences, Kitasato University, Sanriku, Kesen, Iwate 022-01, Japan (早川康博，檑高 二郎: 北里大学水産学部).

*2 Tokyo Agriculture and Forestry Inspection Institute, Konan, Minato, Tokyo 108, Japan (渡辺 悘：東 京農林規格検查所). 


\section{Methods and Materials}

\section{Observation of Movement and Feeding}

Abalones, Haliotis discus hannai, starved previously for a few days, were reared in the tank of development surface $6.48 \mathrm{~m}^{2}(2.4 \mathrm{~m} \times 1.0 \mathrm{~m} \times 0.6 \mathrm{~m}$ depth) without shelters, to which Obs. I referred. Abalone movements in the experimental tanks were observed every 30 minutes for several days in September 1984 (Obs. I-A), and in May 1985 (Obs. I-B). Abalones were reared in the tanks with six U-shaped concrete shelters $(38 \mathrm{~cm} \times$ $20 \mathrm{~cm} \times 20 \mathrm{~cm}$ high), to which Obs. II referred.
Observation was done in September 1984 (Obs. II-A), and in May 1985 (Obs. II-B). Abalones in both Obs. I and Obs. II were not supplied with food during the observation of movement. Means (ranges) of the shell length and the wet weight of abalones used in Obs. I-A were $73.5 \mathrm{~mm}$ (69.9$78.3 \mathrm{~mm})$ and $47.8 \mathrm{~g}(39.7-61.2 \mathrm{~g})$, respectively, Those in Obs. II-A were $74.4 \mathrm{~mm}(70.6-76.6 \mathrm{~mm})$ and $53.0 \mathrm{~g}$ (46.9-57.8 g). In Obs. I-B and Obs. II-B, those were $68.4 \mathrm{~mm}(64.2-75.0 \mathrm{~mm})$ and $41.3 \mathrm{~g} \quad(33.7-60.4 \mathrm{~g})$. Water temperature and light intensity were measured at the time of observation. Moving velocities and relative direc-

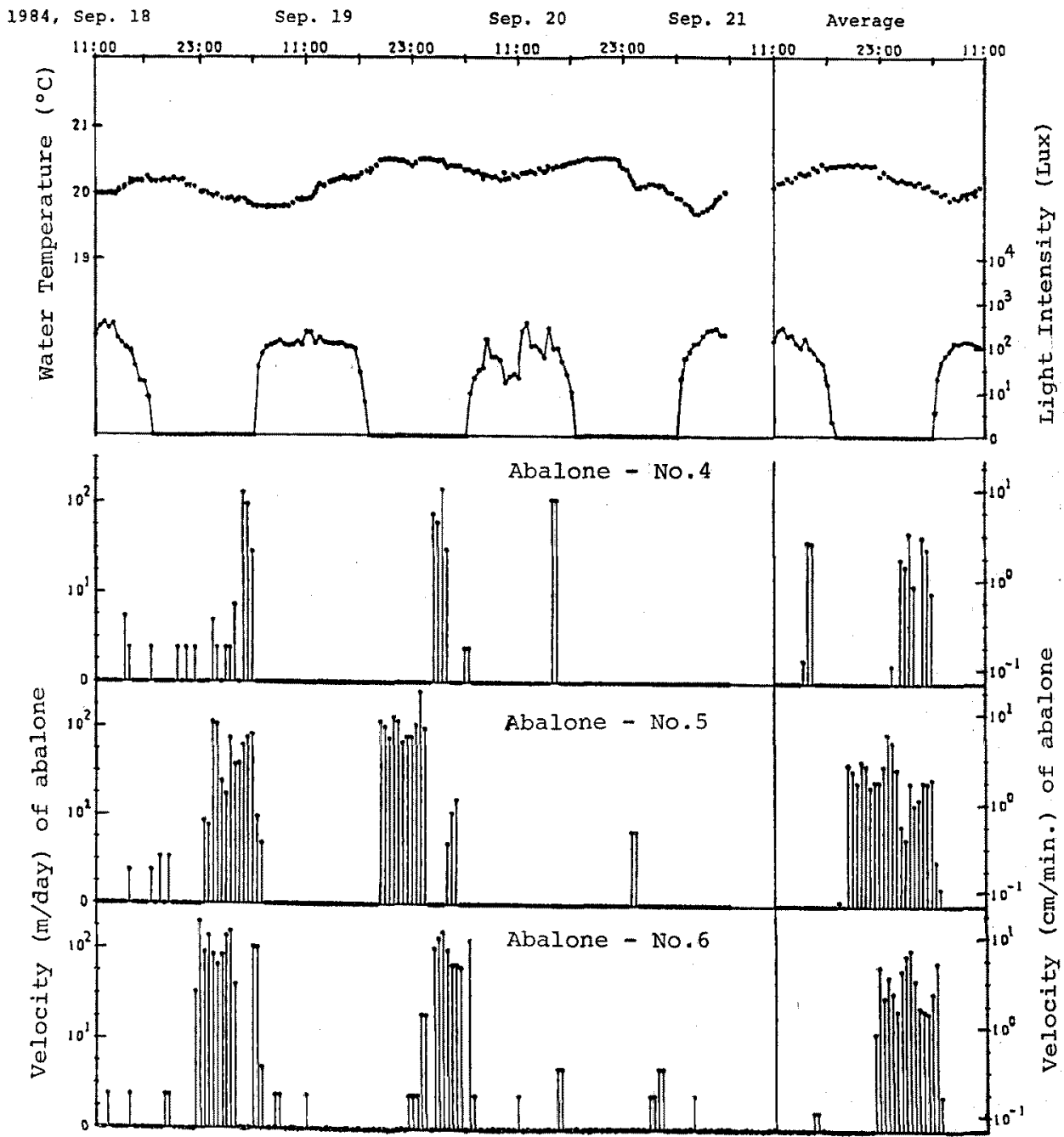

Fig. 1. Velocities of abalones in the experimental tank with neither shelters nor algae (Obs. I-A). Averaged diurnal cycle of velocity are on the right hand. Note that velocity and light intensity are plotted on the logarithmic scale except for zero. Abalone-No. 4, No. 5, and No. 6 had the shell length of $71.3 \mathrm{~mm}$ and the wet weight of $44.6 \mathrm{~g}, 74.8 \mathrm{~mm}-46.9 \mathrm{~g}$, and $78.3 \mathrm{~mm}-$ $61.2 \mathrm{~g}$, respectively. 
tions were calculated from shifts in their locations on the development surface of the experimental tank. The observed data provided a statistics of abalone movement and active duration in a day.

Next, abalones were kept with young brown algae of Undaria pinnatifida distributed uniformly in the same tank without shelter, to which Obs. III referred. Each alga was about $10 \mathrm{~cm}$ long and the wet weight was about $0.7 \mathrm{~g}$. The eaten algae were counted everyday for 25 days from January to February 1985. The experiment was begun using six abalones with initial number of algae at 162. Means (ranges) of the shell length and the wet weight of six abalones used in Obs. III were $73.0 \mathrm{~mm}(70.1-75.6 \mathrm{~mm})$ and $52.8 \mathrm{~g}$ (43.2-58.7 g), respectively. Daily feeding rate (DFR) was calculated as the ratio of algal weight eaten daily to the initial wet weight of abalone. Resultant data were compared with numerical calculations based on the stochastic model as well as the deterministic one.

\section{Modelling}

A deterministic model and a stochastic model were taken into consideration. These were corresponding to Model-II and Model-III in the previous paper, ${ }^{8)}$ respectively. These models consisted of submodels for moving, feeding, and resting behavior of abalones.

In the submodel for moving, abalone's movement was characterized by velocity and relative direction based on the statistics of observed movements in this study.

In the submodel for feeding and resting, hourly feeding rate (HFR) and daily active duration (DAD) in hours formed the very core of feeding speed in a day, since DFR was a product of HFR and DAD. Both HFR and DAD were estimated, based on other published data by Sakai, ${ }^{7 /}$ Kikuchi and $\mathrm{Uki}^{8)}{ }^{8)} \mathrm{Uki},{ }^{97}$ and Uki and Kikuchi. ${ }^{102}$

Of course, the rest of DAD was the daily resting duration, then abalones were assumed to move and feed actively before their own DAD is over. Details of these models would be presented later together with the adopted frequency distributions of parameters in each submodel.

\section{Results}

\section{Observation of Movement and Feeding}

An example of abalone velocity (Obs. I-A) is shown in Fig. 1, where each diurnal cycle is presented by the averages at the same time in a day.
Nocturnal movements are remarkably observed. Abalones sometimes moved around the tank with neither shelter nor food, at the speed of more than ca. $10(\mathrm{~cm} / \mathrm{min})$, but mostly at 1 to $5(\mathrm{~cm} / \mathrm{min})$. Ino $^{11)}$ reported on relatively higher velocity (16.1 $\mathrm{cm} / \mathrm{min}$ ) of this species than others in his pioneering study on abalones. Statistics of velocities are tabulated in Table 1. Since the movements were observed every half an hour, the number of data (1344) in Obs. I, for instance, denotes the total 28 abalone-days. The number of data (1047) in the case of zero velocity indicates the total resting duration, or mean daily resting duration of ca. 18.7 hours, and mean DAD of ca. 5.3 hours. Therefore, mean abalone in Obs. I moved at $3.50 \mathrm{~cm} /$ min) for 5.3 hours, covering $11.1 \mathrm{~m}$ in a day. Abalones moved at a similar speed for shorter periods when with shelters in Obs. II.

Frequency distributions of velocity and relative direction are shown in Fig. 2. The normal and lognormal distributions were tested to be fitted to the observations, using a chi-square test. Only the observed frequency distribution in Obs. II did not differ significantly at $5 \%$ level (the chisquare of 11.9 with 11 degrees of freedom) from the lognormal distribution, that is, the normal distribution of log-transformed velocity with its mean of 0.30 and standard deviation (SD) of 0.44 that were the sample mean and SD. Others were fitted in vain. The lognormal distribution still seemed to be possibly useful for its simplicity in simulating abalone's velocity even if without shelters.

As for relative direction, von Mises (circular normal) distribution ${ }^{122}$ were tested, suggesting an insufficient fitness to observed ones (chi-square values; 70.4 for Obs. I, 29.7 for Obs. II with 11 degrees of freedom in each case). Nevertheless, the von Mises distribution with doubling angles might be applicable to abalone's orientation, because abalones tended to make round trips, as was shown by the observed bimodal frequency distribution of relative directions. The von Mises distribution may be symbolized by $V M(r, i \cdot t)$, where $r$ is the mean vector length, $i \cdot t$ is the mean angle, and $i$ is the number of modes. In Fig. 2, is shown the fitted distribution for Obs. I (Obs. II); $V M(r, 2 \cdot t)$ where $r$ and $t$ are estimated at $0.416(0.403)$ of the sample mean vector length, and at $0.031(-0.010)$ radians of the half of the sample mean angle, respectively.

There is little difference between the frequency distribution of relative directions and that of 
Table 1. Statistics of velocities of abalone Haliotis discus hannai in the experimental tank without foods. $\quad N$ and $n$ denote the number of data. Mean and SD denote the arithmetic mean and the standard deviation, respectively. Log transformation gives the transformation of velocity into the common logarithm

\begin{tabular}{|c|c|c|c|c|c|c|c|c|c|c|c|}
\hline \multirow{2}{*}{\multicolumn{2}{|c|}{ Observations \& Date }} & \multicolumn{3}{|c|}{$\begin{array}{l}\text { Water temperature } \\
\left.\qquad{ }^{\circ} \mathrm{C}\right)\end{array}$} & \multirow[b]{2}{*}{$N=$} & \multirow{2}{*}{$\begin{array}{c}\begin{array}{c}\text { Velo } \\
V=0\end{array} \\
\mathrm{n}=\end{array}$} & \multirow[b]{2}{*}{$\mathrm{n}=$} & \multicolumn{2}{|l|}{$\left.\min _{V>0}\right)$} & \multicolumn{2}{|c|}{$\begin{array}{c}\log \\
\text { transformation } \\
V>0\end{array}$} \\
\hline & & $N=$ & Mean $=$ & $\mathrm{SD}=$ & & & & Mean $=$ & $\mathrm{SD}=$ & Mean $=$ & $\mathrm{SD}=$ \\
\hline \multicolumn{12}{|c|}{ Without shelters } \\
\hline Obs. I-A & $\begin{array}{r}\text { '84, Sep. } 14 \\
\text {-Sep. } 21\end{array}$ & 192 & 19.6 & 1.8 & 1104 & 883 & 221 & 2.97 & 3.68 & 0.047 & 0.664 \\
\hline Obs. I-B & $\begin{array}{rr}\text { '85, May } & 2 \\
- \text { May } & 3 \\
\end{array}$ & 48 & 11.6 & 1.0 & 240 & 164 & 76 & 5.02 & 3.39 & 0.584 & 0.375 \\
\hline Obs. I & & & & & 1344 & 1047 & 297 & 3.50 & 3.71 & 0.184 & 0.647 \\
\hline \multicolumn{12}{|c|}{ With shelters } \\
\hline Obs. II-A & $\begin{array}{r}\text { '84, Sep. } 14 \\
\text {-Sep. } 21\end{array}$ & 192 & 19.4 & 1.1 & 672 & 616 & 50 & 2.64 & 3.15 & 0.233 & 0.405 \\
\hline Obs. II-B & $\begin{array}{r}85, \text { May } 22 \\
\text {-May } 27 \\
\end{array}$ & 144 & 13.2 & 2.8 & 720 & 621 & 99 & 3.33 & 2.91 & 0.332 & 0.459 \\
\hline Obs. II & & & & & 1392 & 1237 & 155 & 3.08 & 3.01 & 0.296 & 0.441 \\
\hline
\end{tabular}
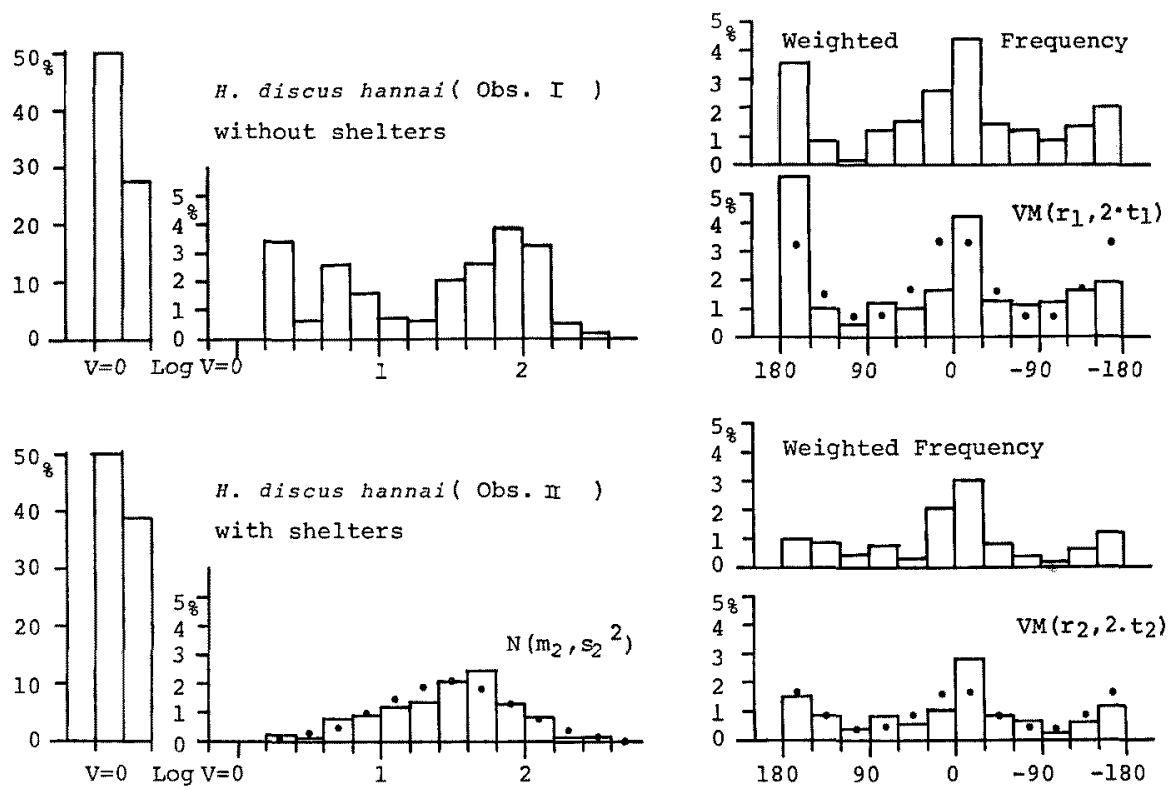

Fig. 2. Frequencies of velocity (left) and relative direction (right) of abalones in the tank without algae (Obs. I and Obs. II). Fitted distributions are shown by dots with their abbreviated formulas, $N$ and $V M$, the normal and the von Mises distributions, respectively. Note that the velocities ( $\mathrm{m} /$ day) of abalones in activity are on the common logarithmic scale, and relative directions are positive anticlockwise.

weighted directions (resultants of velocities and directions), suggesting that abalones have no special directions biased by high-speed movements. So, it is probable that there is no correlation between velocity and relative direction until abalones have perceived their foods.

Results of abalone's feeding upon algae (Obs. III) are to be presented later in comparison with those of stochastic and deterministic calculations. 


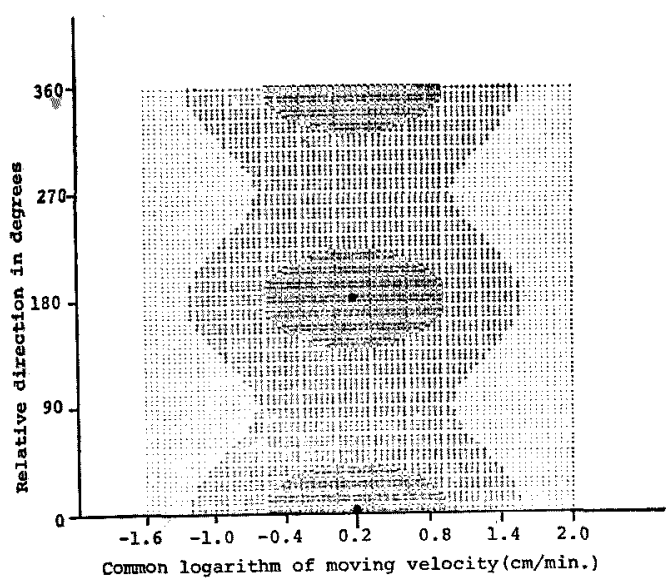

Fig. 3. Joint distribution of log-transformed velocity and relative direction for sinulating abalone's movement. Confidence region for confidence coefficient of $50 \%, 95 \%$ and more than $95 \%$ is shown by darker, dark and grey shade, respectively. Dots indicate the maximum joint probability.

\section{Stochastic Model}

1 Submodel for Movement

To simulate feeding behavior in Obs. III without shelters, results of Obs. I were used as fundamental statistics to estimate parameters for stochastic variables of velocity and relative direction. Therefore, common logarithms of moving velocities were characterized by the normal distribution with its mean of 0.2 and SD of 0.6 , which were approximate value of the sample mean and SD in Obs. I. On the other hand, relative directions were characterized by the von Mises distribution described above for Obs. I. Moreover, these two distribution were assumed independent of each other. The joint distribution of velocity and relative direction is shown in Fig. 3. The confidence regions of this joint distribution was calculted discretely, from -1.6 to 2.0 every 0.05 for log-transformed velocity and from 0 to 360 degrees every 4 degrees for relative direction.

The followings are also assumed: 1) An abalone moves around the restricted tank with a velocity and an orientation which are iteratively given by the above joint distribution every 30 minutes. 2) It never fails to find out algae once encountered within its perceptible diameter (PD), approach them with its mean velocity, and start feeding algae when encountered within its test diameter (TD). 3) The ratio of PD to TD is arbitrarily taken as 1.5, the circle of TD being inscribed in the perceptible circle of PD. 4) An abalone spends its own DAD in any of moving around, approaching and feeding.

In other words, algal distribution can have a decisive effect on abalone's movements, even though they are primarily governed by the joint distribution stated above.

2 Submodel for Feeding and Resting

It is reported by $\mathrm{Uki}^{92}$ that HFR and the feeding

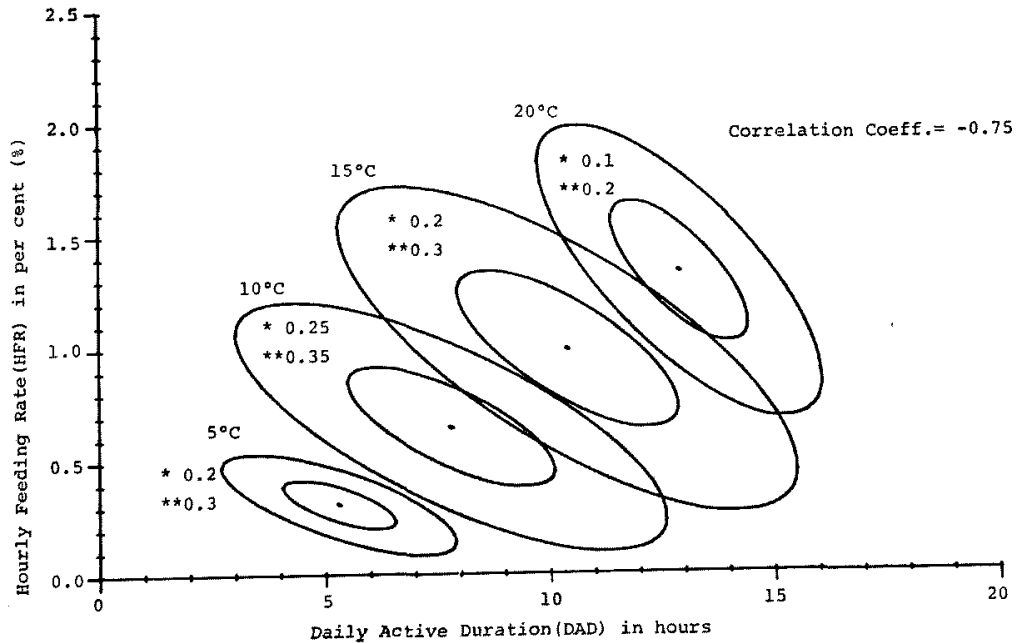

Fig. 4. Joint distribution (bivariate normal distribution) of daily active duration (DAD) and hourly feeding rate (HFR) for simulating abalone's feeding and resting. Confidence regions for $50 \%$ and $95 \%$ of confidence coefficients are given by probability ellipses together with dots of the maximum, where correlation coefficient, and variation coefficients of DAD $(*)$ and HFR(**) are also shown. 
duration increase with higher water temperature, so DFR may be regulated by water temperature. As a matter of fact, HFR and DAD are very variable even if they are measured under the condition of fixed temperature and algal species quality, partly because of uncertain measurement of wet weight, partly because of individual variation in abalones, partly because of other factors than temperature.

In the existing state of things, it might be reasonable to assume that HFR and DAD are stochastic variables dependent on each other. They are characterized by a bivariate normal distribution with a certain negative correlation coefficient in order not to amplify the variance of daily feeding rate too much, as is shown in Fig. 4. Published data $^{7-10)}$ on HFR and DAD were rearranged to introduce simple regressions for estimate of mean $H F R$ and mean DAD;

$$
\begin{aligned}
& \text { mean } H F R(j)=0.068 \cdot T(j)-0.027 \\
& \text { mean } D A D(j)=0.51 \cdot T(j)+2.7
\end{aligned}
$$

where $T(j)$ is water temperature in degrees centigrade at the time of $j$, and units of HFR and DAD are per cent and hours, respectively. Formulas 1) and 2) result in mean $D F R$ of a quadratic function of $T(j)$. This mean DFR is not always compatible with the empirical linear regression, ${ }^{10)}$ but this approximates to the regression under the rather cold temperature $\left(2.4\right.$ to $\left.5.8^{\circ} \mathrm{C}\right)$ of Obs. III. The bivariate normal distribution of DAD and HFR may be symbolized by $B N\left(m x, m y, s x^{2}, s y^{2}\right.$, $c)$, where parameters of $m x, m y, s x, s y$, and $c$ denote mean DAD, mean HFR, SD of DAD, SD of HFR, and correlation coefficient between DAD and HFR, respectively. Once these parameters are given daily, stochastic variables of DAD and HFR are generated.

In generating these variables, correlation coefficient, and variation coefficients of DAD and HFR are tentatively taken as in Fig. 4, because of insufficient experimental data. If a mean $D A D$ and a mean $H F R$ are determined by a water temperature in a given day, the stochastic value of DAD in that day is given by a normal random number, that in turn provides a conditional distribution for the stochastic value of HFR. Thus, the very core of stochastic variables of feeding and resting is characterized by the bivariate normal distribution parameters of which are controlled mainly by water temperature, though the correlation coefficient and variation coefficients might be possibly affected by other factors. DAD and
HFR are given daily, according to the daily mean temperature. The value of DFR based on these DAD and HFR is expected to be the maximum DFR for the given day. Actual DFR based on simulation is usually less than it, because a certain time of DAD is to be spent for abalone's movement.

In simulating abalon's feeding, the followings are assumed: 1) An abalone that encountered living algae within its diameter of TD, starts feeding on them and continues to do so at the speed of its own HFR for that day, as long as its own DAD remains. 2) When DAD is over in the middle course of feeding on alga, feeding is taken over the next night at the same position as last night without searching all over again. 3) After eating up the alga, it immediately starts moving to perceive another alga.

In simulating abalone's resting, the following is assumed: 1) When DAD is over, an abalone suddently stops doing things and there and then rests during the rest of that day, without homing or anything like that.

Although the growth processes of abalones as well as algae might be worth while being considered in another submodel for growth, they are woven here in this submodel. The daily growth rate (DGR) of abalone (the ratio of increased wet weight in a day to the initial wet weight) is assumed to be given by the linear regression of DFR by Sakai. $^{\text {T) }}$ On simulation, an abalone grows up during the resting period, depending on its own DGR derived from actual DFR, while some growth of living algae occurs, following the empirical growth curve of their thalli. Linear relations between wet weight and test diameter of abalone were given by the empirical regression preented by Sakai. ${ }^{13)}$ Those between wet weight and thallus length of algae depended upon another regression by Nonaka and Iwahashi. ${ }^{14}$

\section{Application}

To simulate the abalone feeding on algae in Obs. III, numerical simulation based on this stochastic model with submodels for movement, feeding and resting was made. On simulation, 162 algae of $10.0 \mathrm{~cm}$ and $0.74 \mathrm{~g}$ were grazed by 6 abalones of $7.3 \mathrm{~cm}$ (TD) and $52.8 \mathrm{~g}$ on the same development suface as in Obs. III. Algal growth was taken as a linear one with constant growth rate of $0.03(\mathrm{~cm} / \mathrm{day})$, since the mean length of remaining algae at the end of Obs. III (after 25 days) was found only a slight increase of $0.7 \mathrm{~cm}$. 


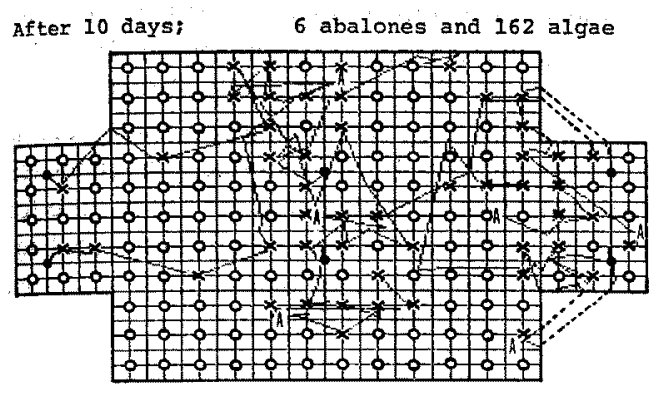

Development surface ( $1 \mathrm{~m} \times 2.4 \mathrm{~m} \times 0.6 \mathrm{~m})$

Fig. 5. Simulation of abalone's feeding upon algae in the restricted area. Living algae, eaten algae, abalones and their initial locations are denoted by open circles, crosses, letter $\mathrm{A}$ and solid circles, respectively.
After 25 days, tentative simulation was made under the assumed water temperature of $5.5^{\circ} \mathrm{C}$.

As a whole, an abalone is to move, approach and feed during its activity in a stochastic fashion while it grows up during resting in a rather deterministic way. An example of stochastic simulation is shown in Fig. 5, where young brown algae have initially been uniformly-distributed. Abalone's grazing seems to make the algal population patchily-distributed.

\section{Deterministic Model}

Abalones behave themselves in such a deterministic fashion that they have only to cover the average area occupied by an alga in order to perceive the algal location, and only to walk the
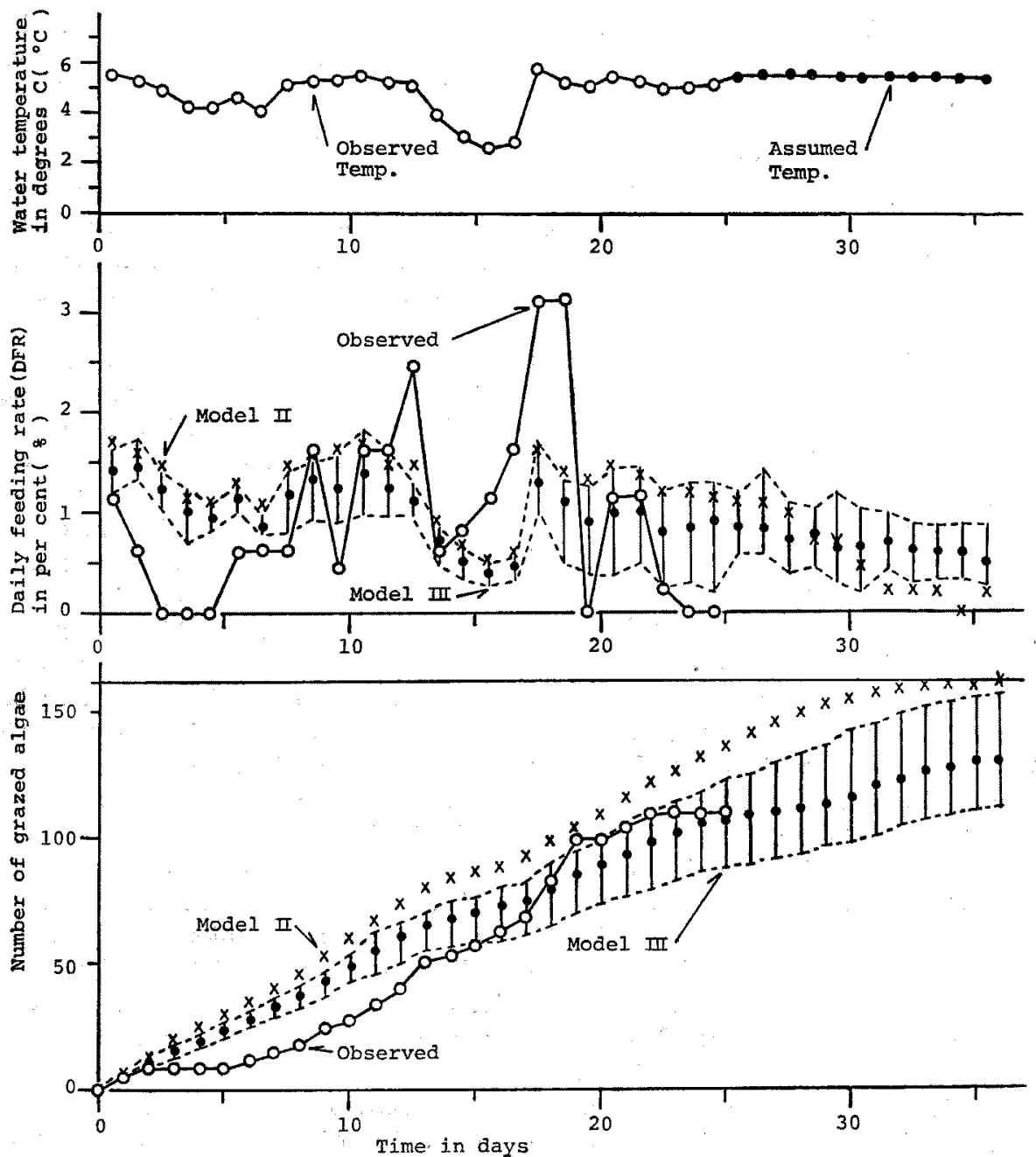

Fig. 6. Comparison of observed feeding (Obs. III) with the deterministic simulation (Model-II) and the stochastic simulation (Model-III). Means of ten calculations are plotted with their ranges in the stochastic simulation. After 25 days, tentative simulations are shown, deriving from assumed water temperatures. 
distance in $\mathrm{Ta}$ straight line between the alga and themselves in order to find the alga. Thus time for finding the $N$-th alga; $T l$ is given by;

$$
\begin{aligned}
T 1= & S /\{(A-N+1) \cdot(P D \cdot V \cdot M)\} \\
& +(P D-T D) /(V \cdot M)
\end{aligned}
$$

where $S$ is feeding area, $A$ is the number of algae at the initial stage, $P D$ is the perceptible diameter, $T D$ is the test diameter, $V$ is the mean velocity, and $M$ is the number of abalones. Time for eating up the $N$-th alga (T2) is given by,

$$
T 2=A W /(M \cdot M W \cdot(\text { mean } H F R))
$$

where $A W$ is the wet weight of an alga, $M W$ is the wet weight of an abalone, and mean HFR is the hourly feeding rate given by formula 1 ). Daily growth of $M W$ as well as $A W$ occurs in the same way as stated in the stochastic model. The sum of $T 1$ and $T 2$ gives total time for eating up the $N$-th alga. When the cumulative sum exceeds mean $D A D$ given by formula 2), feeding on the algae is ceased and the number of eaten algae until then is calculated. Daily iteration of calculation based on formulas 1) to 4) forms the core of this deterministic simulation.

\section{Comparison of Simulation}

Comparison of observed feeding with both the stochastic and deterministic simulation is shown in Fig. 6, where the time changes in the number of eaten algae and the daily feeding rate suggest that the stochastic model may simulate abalone's feeding behavior to a certain extent.

The stochastic simulation is characteristic of expanding range of resultant calculation as time passes, and possible infinite searching for algae when algal population becomes low.

The deterministic simulation indicates a finite time for finishing eating up all algae, and higher daily feeding rate, because of no loss of time for moving around in vain.

Great differences of daily feeding rate between observation and simulation are found at the beginning of experiment when abalones are easily subject to some great stress, and after 15 to 19 days when an abrupt change in water temperature was measured coincidentally.

While little significant difference of growth of each abalone was observed at the end of Obs. III, the most efficient one among simulated abalones gained ca. $1 \mathrm{~g}$ of wet weight, and the most unfortunate one lost ca. $1 \mathrm{~g}$ in the stochastic model. However, these simulated changes in abalone growth were not enough to be commented further.
Under an assumed constant temperature after 25 days, actual DFR in the stochastic simulation as well as the deterministic one, is found decreasing gradually as the remaining algae become sparsely populated. Of course, the simulated abalones in the restricted area can never leave for other areas when the intervals between feedings are beyond their giving-up time (GUT), ${ }^{1-8)}$ if any.

\section{Discussions}

Aggregative distribution of abalones in natural waters in the day time was reported to be characterized by a negative binomial distribution, which suggested the mutual attractive effect of abalones upon the others and the clumped (contagious) distribution of favorable habitats to abalones. ${ }^{15,187}$ On simulation, abalone's locations during resting, equivalent to the habitats reported above, are governed by log-normal random velocities, circular normal random orientations, and algal distribution patterns when perceived or encountered. Thus, the distribution of resting locations would be far from the aggregative one. The perceptible diameter is arbitrarily assumed one and half times as large as the test diameter, partly because of little knowledge on it under any given diffusive condition of causal chemicals, and partly because of a probable short range of effective perception, suggested by the fact that it often took a few days for the starved abalone in the tank to find several adult algae given in satisfaction of their starvation. The model should be developed to include the submodel for abalone's ability of perceiving mutual existence and favorable habitat as well as food.

Above-normal feeding rate after the period of cold temperature in Fig. 6 could be better explained by the concept of catch-up growth ${ }^{17)}$ than by this simulation. On simulation, feeding speed HFR, active duration DAD, and thus, daily feeding rate DFR of individual abalones are controlled by the bivariate normal distribution related exclusively to the surrounding temperature. Feeding activities, however, must be effected by some physiological feed-back system related to assimilation itself. Moreover, the growth process has to be associated with abalone's energy budget; income from assimilation, and outgo derived from basal metabolism and movement. Therefore, submodels have to be developed for feeding based on the physiological feed-back and for growth based on energy budget in abalones.

As for the interspecific relation between abalones 
and algae, it is clear that algal distribution may affect the abalone's feeding behavior and vice verșa, as is suggested in Fig. 5. Most optimal foraging models have been based on the short term goal of maximizing time rate of food intake. An optimal forager, however, could aim to maximize its fitness in the longer term in such an elaborate fashion that relatively slower predators like abalones might adapt themselves better to the natural rocky shores by allowing the algae to flourish there continuously.

With essential and further sophisticated submodels, the stochastic simulation of feeding behavior might be applicable to a quantitative estimate of predator-prey relationship between abalones and algae. Understanding this relationship will be expected to lead not only to aquaculture but also to conservation and utilization of coastal rocky shores.

\section{Acknowledgements}

The authors would like to thank Dr. R. Kado for his encouraging advice, and Mr. K. Nishimura and Mr. K. Yamada, the former students, School of Fisheries Sciences, Kitasato Univ., for their observation work. Experimental abalones were prepared through the courtesy of Iwate Prefectural Mariculture Center. Part of this paper was presented at the symposium (Aquaculture '86) in Trondheim, Norway held by International Federation of Automatic Control. This study was partly supported by Grant-in-aid from the Ministry of Education, Science and Culture, Japan.

\section{References}

1) E. L. Charnov: Theor. Popul. Biol., 9, 129-136 (1976).

2) J. R. Krebs, J. C. Ryan, and E. L. Charnov: Anim. Behav., 22, 953-964 (1974).

3) Y. Iwasa, M. Higashi, and N. Yamamura: Amer. Natur., 117, 710-723 (1981).

4) R. W. Elner and R. N. Hughes: J. Anim. Ecol., 7, 103-116 (1978).

5) R. Kitching: Oecologia, 7, 95-116 (1971).

6) Y. Hayakawa and J. Kittaka: Nippon Suisan Gakkaishi, 50, 233-240 (1984).

7) S. Sakai: Nippon Suisan Gakkaishi, 28, 766-779 (1962).

8) S. Kikuchi and N. Uki: Bull. Tohoku Reg. Fish. Res. Lab., 33, 69-78 (1974).

9) N. Uki: Bull. Tohoku Reg. Fish. Res. Lab., 43, 53-58 (1981).

10) N. Uki and S. Kikuchi: Bull. Tohoku Reg. Fish. Res. Lab., 45, 45-53 (1982).

11) T. Ino: Bull. Tokai Reg. Fish. Res. Lab., 5, 1102 (1952).

12) E. Batschelet: Circular Statistics in Biology, 1st ed., Academic Press, London, 1981, pp. 275-290.

13) S. Sakai: Nippon Suisan Gakkaishi, 28, 899-904 (1962).

14) T. Nonaka and Y. Iwahashi: The Aquiculture, 9 , 229-236 (1962).

15) H. Fushimi and T. Sasaki: Bull. Shizuoka Pref. Fish. Exp. Stn., 11, 1-13 (1977).

16) M. Inoue: The Aquiculture, 20, 147-160 (1972).

17) R.M. Sibly and P. Calow: Physiological Ecology of Animals: an evolutionary approach, Blackwell Sci. Pub., Oxford, 1986, pp. 1-178. 